\title{
Systems approach to monitoring and evaluation guides scale up of the Standard Days Method of family planning in Rwanda
}

Susan Igras, ${ }^{a}$ Irit Sinai, ${ }^{a}$ Marie Mukabatsinda, ${ }^{b}$ Fidele Ngabo, ${ }^{c}$ Victoria Jennings, ${ }^{a}$ Rebecka Lundgren ${ }^{a}$

Scaling-up lessons included: (1) simplifying provider training and client materials; (2) ensuring core aspects of the intervention, for example, that the CycleBeads client tool was integrated into the supply chain system; (3) addressing provider-generated medical barriers; and (4) managing threats from changing political and policy environments. A focus on systems, the use of multiple M\&E data sources, maintaining fidelity of the innovation, and ongoing environmental scans facilitated scale-up success.

\begin{abstract}
There is no guarantee that a successful pilot program introducing a reproductive health innovation can also be expanded successfully to the national or regional level, because the scaling-up process is complex and multilayered. This article describes how a successful pilot program to integrate the Standard Days Method (SDM) of family planning into existing Ministry of Health services was scaled up nationally in Rwanda. Much of the success of the scale-up effort was due to systematic use of monitoring and evaluation (M\&E) data from several sources to make midcourse corrections. Four lessons learned illustrate this crucially important approach. First, ongoing M\&E data showed that provider training protocols and client materials that worked in the pilot phase did not work at scale; therefore, we simplified these materials to support integration into the national program. Second, triangulation of ongoing monitoring data with national health facility and population-based surveys revealed serious problems in supply chain mechanisms that affected SDM (and the accompanying CycleBeads client tool) availability and use; new procedures for ordering supplies and monitoring stockouts were instituted at the facility level. Third, supervision reports and special studies revealed that providers were imposing unnecessary medical barriers to SDM use; refresher training and revised supervision protocols improved provider practices. Finally, informal environmental scans, stakeholder interviews, and key events timelines identified shifting political and health policy environments that influenced scale-up outcomes; ongoing advocacy efforts are addressing these issues. The SDM scale-up experience in Rwanda confirms the importance of monitoring and evaluating programmatic efforts continuously, using a variety of data sources, to improve program outcomes.
\end{abstract}

\section{BACKGROUND}

R wanda is the most densely populated country in Africa and one of the poorest countries in the world. ${ }^{1}$ Following the devastating 1994 genocide, the country made intensive efforts to improve its social, economic, and health conditions. But almost 2 decades later, the health system still faces many challenges, including meeting people's reproductive health needs.

\footnotetext{
aGeorgetown University's Institute for Reproductive Health, Washington, DC, USA.

${ }^{\mathrm{b}}$ Institute for Reproductive Health, Kigali, Rwanda.

'Ministry of Health [Rwanda], Kigali, Rwanda.

Correspondence to Rebecka Lundgren (lundgrer@georgetown.edu).
}

In 2005, the total fertility rate was more than 6 children per woman, and almost $40 \%$ of women of reproductive age had an unmet need for modern contraceptive methods. $^{2}$

In an effort to help women meet their contraceptive needs and achieve healthy timing and spacing of pregnancies, the Rwanda Ministry of Health $(\mathrm{MOH})$ joined in partnership with the Institute for Reproductive Health (IRH) at Georgetown University, as well as with other private and faith-based health groups, to expand access to the Standard Days Method (SDM) throughout the country.

SDM is a fertility awareness-based method of family planning based on a woman's menstrual cycle (Box). 


\section{Box. What is the Standard Days} Method?

The Standard Days Method (SDM) is a simple, fertility awareness-based method of family planning developed and tested by Georgetown University's Institute for Reproductive Health. Based on reproductive physiology, SDM identifies the days in the menstrual cycle (days 8-19) when a woman can get pregnant if she has unprotected sex. CycleBeads, a color-coded string of beads, helps women track the days of their cycles when they are most likely to get pregnant. The method works best for women with cycles that usually range 26-32 days. Over half of women meet this criterion.

If the woman does not want to get pregnant, she and her partner avoid unprotected sex on days 8 through 19 of her cycle. An efficacy study found a failure rate for SDM of 5 per 100 woman-years when used correctly. The failure rate during typical use is 12 per 100 woman-years. ${ }^{3}$

SDM has been introduced and assessed in different facility and community-based service delivery settings for over 12 years. ${ }^{4}$ The U.S. Agency for International Development and the World Health Organization have globally recognized the method as a modern, evidence-based contraceptive practice, ${ }^{5}$ and it is currently offered in more than 30 countries.

Because SDM is a low-cost fertility awarenessbased method with no side effects, was acceptable to faith-based groups, and does not require followup visits or resupplies, it filled a special niche in the Rwandan family planning program.

The method had been integrated successfully into both clinical and community-based government services in pilot programs. Scaling up the pilot program to the entire country, however, was a complex task. International family planning research shows that unless a new method is introduced in a systematic and strategic way, results are not likely to be positive or sustainable. ${ }^{6,7}$ For scale up to be successful, understanding the changing environmental contexts in expanded geographic areas-which may differ in significant ways from the pilot sites-is critical, $^{8}$ and the concerns of many key stakeholders must be addressed. ${ }^{9}$ Partner organizations are essential to expand access and to leverage technical and financial resources, but they often have different project and funding durations from the scale-up program.

Developing workforce capacity to offer the new family planning method as part of routine service delivery is at the heart of scaling up, but it takes multiple family planning actors to make this happen, each with varying roles, abilities, and resources to apply to the scale-up process. New methods must be included in supply chain systems, and it can take several years before changes become operationalized in periphery services. Budget allocations for a new method require advocacy and evidence to reassure policy makers during scale up that the program investment is worthwhile. New methods are not yet wellintegrated into routine monitoring and evaluation (M\&E) systems in the early stage of scale up, so additional information sources are required to monitor the pace of expansion and integrity of the innovation. Thus, the process of wide-scale integration of the new method within a complex health system cannot be controlled or monitored to the same extent as more localized introduction efforts during the pilot stage.

To inform our scale-up process, we adopted the principles of the World Health Organization (WHO)/ExpandNet conceptual framework for sustainable scale up, ${ }^{10}$ the corollary Nine-Step Guide to develop a strategic scale-up plan, ${ }^{11}$ and related guidance by Simmons and Shiffman, ${ }^{12}$ who summarize the characteristics of a good scaling-up strategy, based on diffusion of innovation theory ${ }^{13}$ and other literature on scaling up health practices. Such characteristics comprise:

- An intervention that can be adapted to fit into the existing health system

- A participatory approach that includes local and central stakeholders and policy makers

- Reliance on systematic use of evidence for decision-making

- An ongoing focus on sustainability

After providing a brief introduction about the outcomes of the pilot phase as well as goals and outcomes of national scale up, this article provides lessons learned about how to successfully scale up health interventions. These lessons demonstrate the importance of ongoing monitoring and evaluation efforts for making midcourse corrections that support successful scale up.

\section{The Standard \\ Days Method is an inexpensive, fertility awareness-based method with no side effects, and it does not require follow-up visits or resupplies.}

\section{It takes time to integrate new contraceptive methods into routine M\&E systems, so additional data sources are often needed to monitor scale up.}


FIGURE. SDM Program Milestones and Data Collection Timeline, Rwanda, 2002-2012

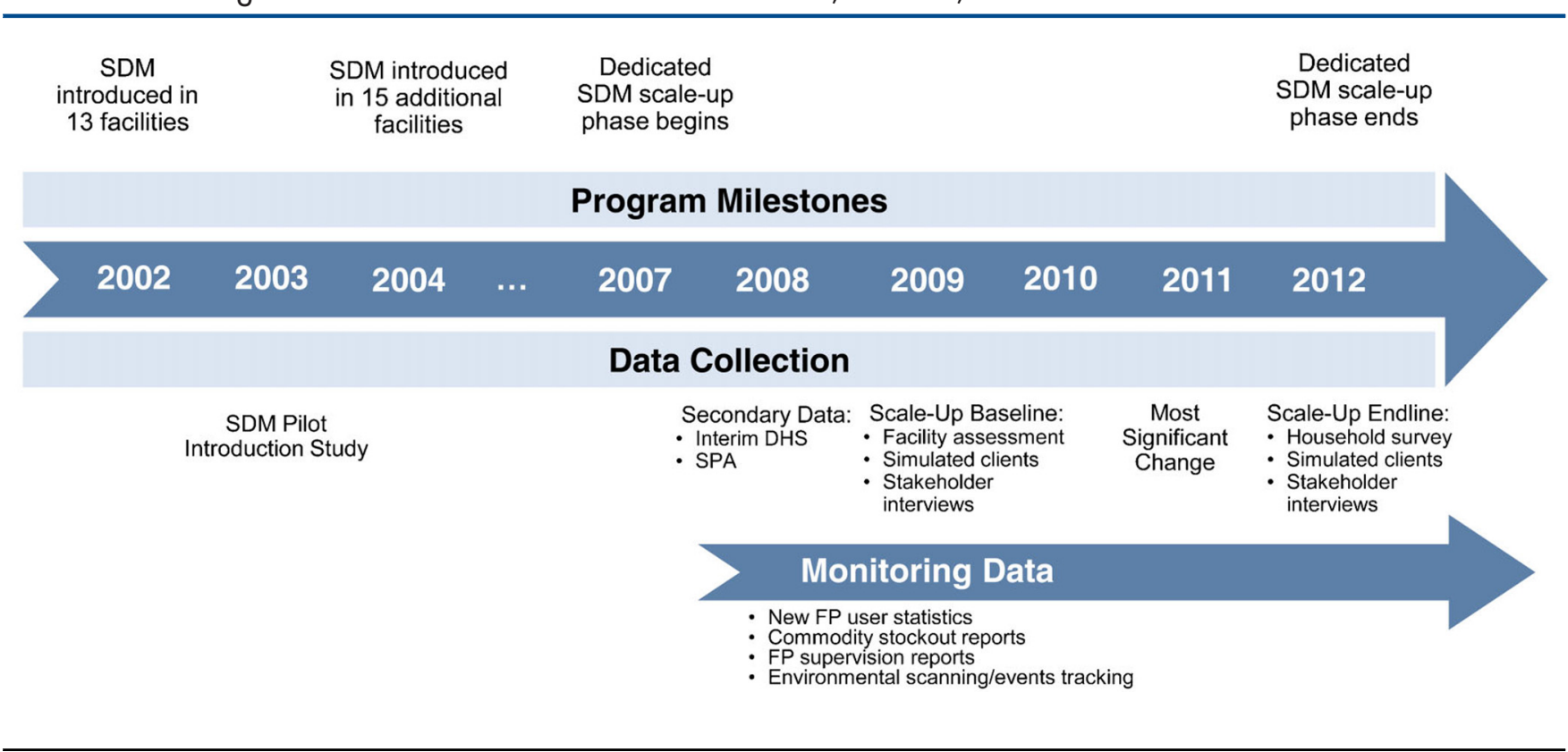

Abbreviations: DHS, Demographic and Health Survey; FP, family planning; SDM, Standard Days Method; SPA, Service Provision Assessment.

During the pilot phase, $23 \%$ of new contraceptive method users chose the Standard Days Method.

\section{SDM INTRODUCTION AND SCALE UP IN RWANDA}

\section{Pilot Phase Demonstrates Demand for SDM}

In 2002, we introduced SDM in Rwanda through a pilot program in 7 public health facilities, 5 clinics run by faith-based organizations, and 1 nongovernmental organization site. In 2004, we introduced SDM in 15 more facilities (Figure).

The pilot program generated substantial demand for SDM: service statistics showed that $23 \%$ of new method users chose SDM. ${ }^{14}$ Interviews and focus groups confirmed that the method was easy to offer by providers, was a viable choice for many couples, and was often adopted by women who had never before used a modern method. Offering SDM also had an additive effect on contraceptive prevalence rates, ${ }^{14}$ making it an attractive option for the Rwandan national family planning program.

\section{Scale-Up Challenges and Goals}

Between 2005 and 2007, the country revitalized family planning efforts, and the $\mathrm{MOH}$ took this opportunity to integrate SDM into the new family planning policies, norms, training curricula, and management information and logistics systems.
Within this favorable policy environment, geographic expansion of SDM services continued in 2007 under a 6-year, dedicated scale-up program. Considerable progress had been made already in both horizontal scale up (geographic expansion) and vertical scale up (institutionalization, such as, inclusion in norms, training, supervision, procurement, and reporting systems). But much work remained:

- The program had to expand to the many districts where SDM was not yet available and build the capacity of national and local organizations to offer the method without outside technical assistance.

- SDM had to be integrated into preservice training - a key element of sustainability.

- The revised family planning policies had to be operationalized so that CycleBeads, a tool to help women track their fertile and infertile days, and related instructional materials would be included in supply chains, and so that SDM would become part of routine service statistics.

- Even though there was top-level approval, scale up required advocacy to create support among policy makers and service providers at 
different levels for adding a new family planning method.

- Scale up also relied on mass media and community-level promotion to ensure potential clients knew of the new method option, its unique attributes compared with other methods, and where to find facilities that offered it.

The Rwanda MOH continued its close involvement with SDM scale up throughout the country via the Maternal and Child Health Task Force and its subsidiary Family Planning Technical Committee, made up of key family planning actors including $\mathrm{MOH}$, donors, and international and national nongovernmental and faith-based organizations. End-of-project goals identified by partners and key stakeholders included:

1. Availability of SDM in $95 \%$ of public and private health facilities that offer family planning and in all community-based family planning services

2. Institutionalization of SDM into family planning support systems

To manage the complex set of actions required, the partners developed a strategic plan to achieve these goals over 6 years, which encompassed strategic planning and coordination of organizational roles, phased-in implementation of activities, MEE, and midcourse corrections throughout the process.

\section{Scale-Up Outcomes}

The dedicated scale-up effort using a systems lens led to near-nationwide availability of SDM by the end of the scale-up period. In fact, by the end of the scale-up project, 717 service delivery points included SDM in the method mix, surpassing the benchmark of 690, and more than 7,000 individuals had been trained to counsel clients on how to use SDM (Table 1). According to endline survey results, awareness of the method among women and men was on the same level as other, more established methods, and $7.4 \%$ of women using family planning chose to use SDM. Most women using SDM at the time of the survey were satisfied with the method $(97.5 \%)$ and planned to continue using it $(87.4 \%)$.

\section{LESSONS LEARNED FROM MONITORING AND EVALUATING THE SCALE-UP PROCESS}

Because scale up is a non-linear process that occurs within complex systems with engagement

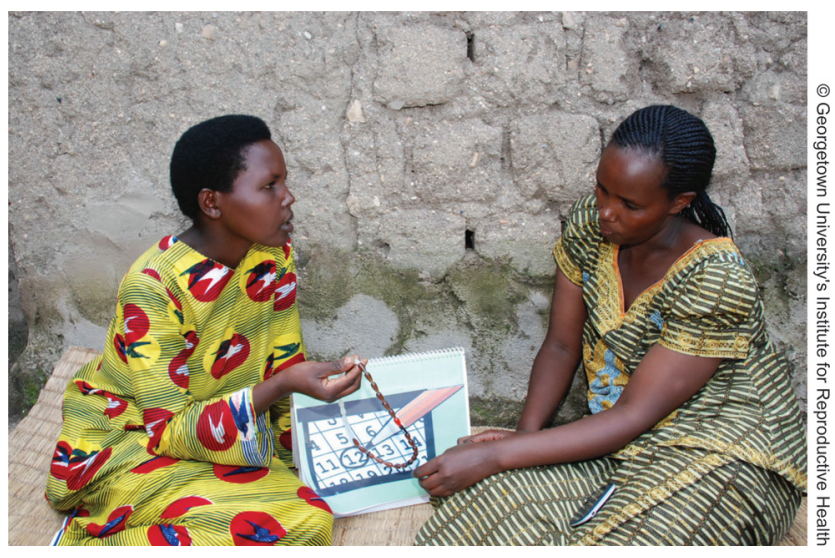

A health care provider shows a client how to use the Standard Days Method of family planning with CycleBeads.

of multiple organizations and health system actors, strategic use of data from multiple sources throughout the scale-up process provides timely information to allow program corrections and to support the policy process. To provide useful information, our M\&E efforts had to cut across multiple levels, sources, and phases (Table 2). Lessons learned about our scale-up process follow, demonstrating the importance of collecting and using data to make midcourse corrections that supported successful scale up.

\section{Lesson Learned 1. Expect to simplify elements of the intervention - even if they worked in the pilot phase - to function at scale and to ensure sustained integration into existing systems.}

Results of provider supervision and client followup visits revealed that providers and clients at the scale-up sites found the training protocols and client materials from the pilot phase too difficult to use. We realized that the SDM intervention needed to be simplified further to support its integration into the national family planning program, since we could not provide the same concentrated attention to the larger number of facilities and community settings as we did to the smaller number of pilot sites.

We then field-tested the resulting simplified user instructions, translated into Kinyarwanda (the native language in Rwanda), to ensure that providers counseled accurately and that clients received correct information using the modified instructions. Client materials were modified a second time in preparation for including SDM in
Training protocols and counseling materials had to be simplified for national scale up. 
TABLE 1. First-Year and End-of-Project Outcomes Compared With Benchmarks

\begin{tabular}{|c|c|c|c|c|c|}
\hline \multirow[b]{2}{*}{ Indicator } & \multicolumn{2}{|c|}{ First Year $(2007)^{a}$} & \multicolumn{2}{|c|}{ End of Project (2012) } & \multirow[b]{2}{*}{$\begin{array}{l}\text { 5-Year } \\
\text { Benchmarks }\end{array}$} \\
\hline & No. & $\begin{array}{c}\% \text { of } \\
\text { Benchmark }\end{array}$ & No. & $\begin{array}{c}\% \text { of } \\
\text { Benchmark }\end{array}$ & \\
\hline Service delivery points that include SDM in the method mix & 356 & 51.2 & 717 & 103.9 & 690 \\
\hline Individuals trained to counsel clients on how to use SDM & 1,679 & 31.0 & 7,472 & 138.4 & 5,400 \\
\hline $\begin{array}{l}\text { Organizations that have capacity to undertake SDM } \\
\text { activities }\end{array}$ & 5 & 50.0 & 7 & 70.0 & 10 \\
\hline $\begin{array}{l}\text { Essential or key policies, norms, guidelines, and protocols } \\
\text { in which SDM is included }\end{array}$ & 2 & 50.0 & 3.5 & 87.5 & 4 \\
\hline $\begin{array}{l}\text { Public or private training organizations that include SDM } \\
\text { in their preservice training and/or continuing education }\end{array}$ & 5 & 100.0 & 5 & 100.0 & 5 \\
\hline $\begin{array}{l}\text { Public or private training organizations that include SDM } \\
\text { in their in-service training }\end{array}$ & 4 & 40.0 & 7 & 70.0 & 10 \\
\hline $\begin{array}{l}\text { Information, education, and communication activities, } \\
\text { materials, and mass media that include SDM }\end{array}$ & 7 & 58.3 & 12 & 100.0 & 12 \\
\hline
\end{tabular}

\section{Problems with integrating CycleBeads into the supply chain affected SDM availability and use.}

social marketing within private-sector pharmacies and clinics.

\section{Lesson Learned 2. Maintain integrity of core aspects of the innovation package.}

M\&E efforts also exposed the importance of defining the intervention "package" clearly-in terms of ensuring both successful scale up and accurate assessments of availability of the package. Although some components of pilot projects must be adapted as mentioned under the first lesson learned, critical aspects of the intervention must remain intact for scale up. According to the partners' definition, the core SDM package included CycleBeads (offered in a small plastic bag with instructions and a multi-year calendar), training curricula and in-service training materials for health care providers and supervisors, and awareness-raising materials and activities that focused on both men and women.

Assessment of data from multiple sources, including ongoing program monitoring data as well as national health facility and populationbased surveys, revealed seemingly incompatible data findings about SDM availability in facilities and use among women. As it turned out, the national surveys used a different definition of the full SDM package, which made a substantial difference in SDM availability and use.

Specifically, according to the preliminary Rwandan Service Provision Assessment (SPA) issued in $2008,{ }^{15} 75 \%$ of facilities that offered family planning reported offering SDM-25\% more than our scale-up monitoring data had indicated. However, interim Demographic and Health Survey (DHS) ${ }^{16}$ data found that while $64 \%$ of women had heard of SDM, only $0.3 \%$ of women said they were using it (Table 3). So although most facilities were seemingly offering SDM and most women had heard of the method, very few women were actually using it.

During the pilot phase, once women had become aware of SDM, there was sizable demand for it; $23 \%$ of new family planning users had chosen SDM during the pilot phase. Although method uptake is expected to be somewhat lower in scaleup sites than in pilot sites, and DHS included sites where SDM had not yet been introduced, the extremely low $0.3 \%$ user figure coupled with the seemingly high percentage of facilities offering the method signaled that something was wrong.

The SPA final report revealed that while $75 \%$ of facilities reported that SDM was available, CycleBeads were observed in only $12 \%$ of facilities-in reality, rendering the method 
TABLE 2. Monitoring and Evaluation Data Collection by Scale-Up Indicator

\begin{tabular}{|c|c|c|c|c|}
\hline Indicator & M\&E Method ${ }^{a}$ & Type of Data & Main Purpose & Timing \\
\hline Outcomes & Household survey & Quantitative & Evaluation & Endline \\
\hline - Awareness and use of SDM & Service statistics & Quantitative & Monitoring & Monthly \\
\hline - Availability of quality services & $\begin{array}{l}\text { "Most Significant Change" } \\
\text { story collection }\end{array}$ & Qualitative & Evaluation & Year 4 \\
\hline \multirow[t]{2}{*}{ - Provider competency } & $\begin{array}{l}\text { Provider supervision and } \\
\text { client follow-up reporting }\end{array}$ & Quantitative & Monitoring & Ongoing \\
\hline & Simulated clients study & Quantitative & Evaluation & Baseline and endline \\
\hline Outputs & $\begin{array}{l}\text { Facility/service delivery } \\
\text { point survey }\end{array}$ & Mixed & Evaluation & Baseline \\
\hline - Providers trained & Stakeholder interviews & Qualitative & Evaluation & Baseline and endline \\
\hline - Clinics offering SDM & Benchmark reporting & Quantitative & Monitoring & Semiannually \\
\hline \multicolumn{5}{|l|}{$\begin{array}{l}\text { - Demand-oriented Information, } \\
\text { Education and Communication (IEC) }\end{array}$} \\
\hline \multicolumn{5}{|l|}{ - Supportive partners/stakeholders } \\
\hline \multicolumn{5}{|l|}{ - Systems integration } \\
\hline Process & $\begin{array}{l}\text { Staff assessments of data on } \\
\text { scale-up status }\end{array}$ & Qualitative & Monitoring & Annually \\
\hline - Scale-up strategy & $\begin{array}{l}\text { Organizational capacity } \\
\text { assessments }\end{array}$ & Qualitative & Evaluation & Ongoing \\
\hline - Dissemination and advocacy & $\begin{array}{l}\text { Environmental scanning, } \\
\text { including key events timeline } \\
\text { reporting }\end{array}$ & Qualitative & Monitoring & Ongoing \\
\hline \multicolumn{5}{|l|}{$\begin{array}{l}\text { - Organizational capacity-building } \\
\text { process }\end{array}$} \\
\hline \multicolumn{5}{|l|}{ - Resource mobilization } \\
\hline - Environmental influences & & & & \\
\hline
\end{tabular}

unavailable in most facilities per the program's definition. In the SPA report, SDM method provision was probably defined as having trained providers at the facility and/or having the method listed in the facility service statistics, without considering actual availability of CycleBeads and other package components.

In 2008, the MOH and the Maternal and Child Health Task Force acted on this evidence by tasking the DELIVER Project (a USAIDfunded project supporting contraceptive supply systems) to address CycleBeads stockouts at facility levels. The DELIVER Project reviewed the mechanism used by health facilities to order contraceptive supplies (including CycleBeads), instituted a new procedure for requesting urgent supplies, and trained health centers and district pharmacists on contraceptive resupply, particularly for new, underused methods. The scale-up resource team became more vigilant in monitoring stockouts in collaboration with DELIVER Project staff. 
TABLE 3. Contraceptive Availability in Facilities Offering Family Planning and Knowledge and Use Among Married Women of Reproductive Age

\begin{tabular}{|c|c|c|c|c|c|}
\hline \multirow[b]{2}{*}{ Method } & \multicolumn{2}{|c|}{ Contraceptive Availability in Facilities ${ }^{a}$} & \multicolumn{3}{|c|}{ Contraceptive Knowledge and Use ${ }^{b}$} \\
\hline & $\begin{array}{l}\% \text { Offer } \\
\text { Method }\end{array}$ & $\begin{array}{l}\text { \% Method Available } \\
\text { on Day of Survey }\end{array}$ & $\begin{array}{l}\text { \% Know of } \\
\text { Method }\end{array}$ & $\begin{array}{l}\text { \% Ever Used } \\
\text { Method }\end{array}$ & $\begin{array}{l}\text { \% Currently } \\
\text { Using Method }\end{array}$ \\
\hline Standard Days Method & 75 & 12 & 64.1 & 1.4 & 0.3 \\
\hline Female sterilization & 6 & & 77.0 & 0.7 & 0.7 \\
\hline Male sterilization & 4 & & 56.0 & 0.2 & 0.1 \\
\hline Pills & 93 & 71 & 89.1 & 15.2 & 6.4 \\
\hline Intrauterine devices & 20 & 44 & 54.4 & 0.8 & 0.2 \\
\hline Injectables & 93 & 71 & 91.3 & 26.1 & 15.2 \\
\hline Implants & 51 & 49 & 57.8 & 2.1 & 1.6 \\
\hline Male condoms & 91 & 69 & 98.4 & 5.9 & 1.9 \\
\hline Female condoms & 35 & 57 & 60.2 & 0.2 & 0 \\
\hline $\begin{array}{l}\text { Emergency contraceptive } \\
\text { pills }\end{array}$ & 16 & 22 & & & \\
\hline
\end{tabular}

About 1 year later, we conducted a facility assessment, in part to determine whether midcourse corrections to the supply chain had resolved the issue with stockouts. The results were encouraging: $90 \%$ of facilities offered SDM and only $8 \%$ experienced stockouts of CycleBeads in the 3 months preceding the survey (Table 4 ).

\section{Lesson Learned 3. Track and address provider performance to avoid unnecessary medical barriers and ensure fidelity of new method protocols at scale.}

A family planning innovation can also lose fidelity during scale up from provider bias and medical barriers. Integration of a new method in a service delivery system requires that providers not only are trained to offer the method but also appreciate its added value, since the providers must adjust their services to include the new method in their program.

During the SDM pilot program, it became clear that many providers doubted whether a fertilityawareness method could be effective. Perhaps in an effort to increase efficacy, some providers applied eligibility criteria that were neither part of the SDM service delivery protocol nor of evidence-based practice, which made the method less accessible. Specifically, some providers required women to monitor their cycle length for several months prior to initiating SDM; to be menstruating at the time they begin using the method; or to have their partner present during the counseling session.

Supervisors corrected such practices during the pilot phase, but this was not feasible during scale up. Early in the scale-up process, $\mathrm{MOH}$ district supervision reports provided observational and anecdotal evidence of alterations in the SDM service-delivery protocol. But to document and better define the existence of barriers to SDM adoption in routine service settings, we conducted a special simulated client study in conjunction with the 2009 facility assessment (mentioned under the second lesson learned). The simulated client study was conducted in facilities where providers were not interviewed for the facility assessment.

Simulated clients were women trained to play the role of clients seeking family planning services. They used specially designed client profile scripts that included contraceptive history, partner relationship, and method preference. After each 
TABLE 4. Results From the Rwanda SDM Scale-Up Facility Assessment and Simulated Client Study, April 2009

\begin{tabular}{lc} 
Facility Audit ( $\mathbf{N}=118$ facilities) & $\%$ \\
\hline Facilities in which the program manager said that SDM was offered & 89.9 \\
Facilities with health providers trained to offer SDM & 94.1 \\
Facilities in which CycleBeads were available on day of audit & 94.0 \\
Facilities experiencing stockouts of SDM in the 3 months prior to the audit & 7.6 \\
Provider Interviews ( $\mathbf{N}=155$ providers) & $\%$ \\
\hline Trained providers that demonstrated correct knowledge of SDM (on 4 key indicators) & $78.0-97.2$ \\
Trained providers who offered SDM to at least 1 client in the 3 months preceding the interview & 90.8 \\
Simulated Clients (N = 28 simulated client visits) & $\%$ \\
\hline Received SDM counseling during the visit & 78.6 \\
Received CycleBeads during the visit & 75.0 \\
Correctly screened for cycle regularity & 81.8 \\
\hline Abbreviation: SDM, Standard Days Method. &
\end{tabular}

clinic visit, the simulated clients completed a checklist about their experience that included more than 80 objective yes/no indicators regarding what should be included in quality counseling in general, and in counseling on SDM in particular. Items included eligibility screening for using the method, mechanisms of action, use of CycleBeads, correct condom use (for those who wished to use condoms on their fertile days), couple communication about the fertile days, and follow up if there were any problems. This methodology had been validated in a number of previous studies. ${ }^{17}$ To respect principles of informed consent in research, providers in 28 selected facilities from the random sample of facilities participating in the facility assessment consented to be visited by a simulated client sometime over the next year, without knowing the specific date of the visit.

The facility assessment found that $94 \%$ of facilities had providers trained to offer SDM, and $94 \%$ had CycleBeads in stock. However, the simulated client study showed clearly that providers were creating unnecessary medical barriers to SDM use, thus diminishing method integrity and availability. For example, $21 \%$ of simulated clients were not offered SDM despite having the appropriate profile for the method (Table 4). One client who received SDM counseling did not receive CycleBeads at the time of her visit; the provider told her to return when her period started. Others who were given information but not counseling about SDM were also told to return when they got their period or to return with their partner so he could be present for the counseling. Moreover, one provider told a client that she did not offer SDM to her clients because she did not trust the method.

The MOH's Maternal and Child Health Task Force and the Family Planning Technical Committee addressed these issues through refresher training and revised supervision protocols in the remaining years of the scale-up process. $\mathrm{MOH}$ supervisors worked with providers to become comfortable with offering the new method, including addressing questions of method effectiveness and reducing medical barriers. A small internal study ${ }^{18}$ conducted in 2011 evaluated the effectiveness of the focused supervision approach and found significant improvement. This improvement was confirmed by later supervision visits around the time of the endline evaluation for the scale-up project.

\section{Lesson Learned 4: Regularly scan, identify, and address changing environmental} influences on scale up.

Since scale-up processes operate within the complex systems in which family planning services are embedded, it is critical to scan environmental
During scale up, providers were imposing unnecessary medical barriers. 


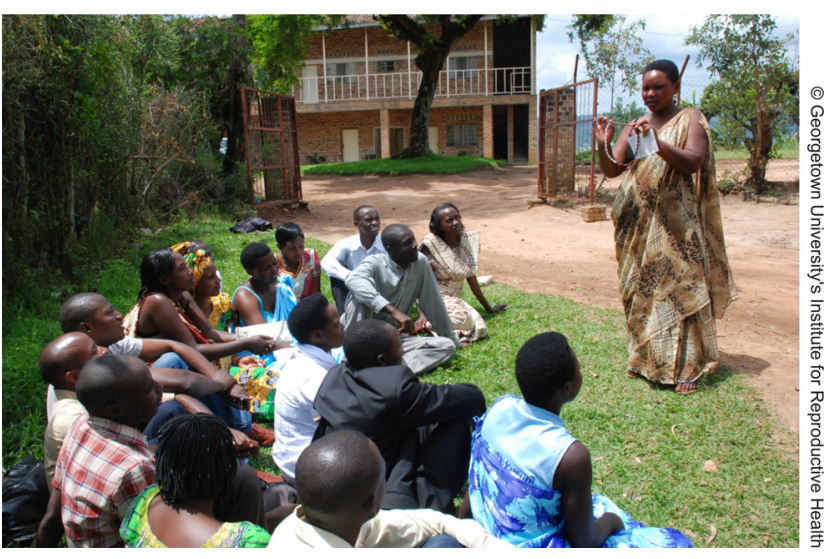

A trainer teaches a group of health care providers how to use CycleBeads, the color-coded string of beads used with the Standard Days Method of family planning.

The government's new focus on longacting and permanent methods tended to divert attention from SDM. a host of sources. Therefore, we collected data on factors that may be influencing scale up, such as changes in national leadership or a family planning trend that becomes apparent only through repeated discourse. ${ }^{19}$

A cross-country analysis of factors influencing scale up of SDM in Rwanda and 4 other countries (Democratic Republic of Congo, Guatemala, India [State of Jharkhand], and Mali) revealed the importance of the political and health policy environments; such factors are not typically identifiable via routine monitoring systems because they are often unexpected, imprecise, and come from environmental factors through other methods including:

- Informal environmental scans to obtain information on social, economic, political, and policy changes but in relatively unstructured ways

- Interviews with staff and scale-up partners to explore their knowledge of the political and policy environments within and external to the national family planning program; this became a regular source of data collection

- Key events timeline, updated semiannually, to track important changes and stakeholder interviews

- FP stakeholder interviews gathered perceptions of forces and factors that might affect scale up from politically connected experts

Assessments from these data sources confirmed that SDM scale up benefited from the Rwandan government's vision of family planning as a crucial national development tool. However, they also revealed the existence of counterforces. In particular, government policy discourse during the scale-up period focused heavily on long-acting and permanent methods which tended to divert attention from SDM. Also, data from environmental scans picked up changes in health financing policies during the second year of scale up. The $\mathrm{MOH}$ began promulgating a health-sector performance-based financing (PBF) system about the same time that scale up of SDM was progressing. The system provided incentives for wellperforming health centers based on the quantity and quality of specific services they delivered, and while SDM was added to the system in 2009, it was dropped in 2010. Essentially, providers had financial incentives to offer other modern methods but not SDM, thus challenging sustainability of the method.

In response to these environmental obstacles, we positioned SDM among policy makers and influential technical stakeholders as a contraceptive option with unique attributes that filled an important niche in family planning programs. It is a long-acting method since clients can and do continue to use the method for years, ${ }^{20}$ it helps to involve male partners, and it increases women's empowerment through basic understanding of their fertility. In addition, we began one-on-one advocacy efforts with individuals who were influential within the PBF Unit and technical arms of the $\mathrm{MOH}$ to provide sound rationales for including SDM in the PBF system. As the 6-year scale-up period ended, this critical issue for sustainability was still unresolved. However, champions had been identified to press the issue further on policy and technical grounds, and it appeared on the way to resolution.

\section{CONCLUSION}

SDM scale up is continuing in Rwanda, as it is in other countries, and the Maternal and Child Health Task Force and other family planning actors are organized to ensure sustainability of method integration.

M\&E from multiple sources, including routine monitoring data and impact evaluations as well as special studies and national surveys, played a critical role in scale up by providing timely information for evidence-based decision-making and midcourse corrections to address a number of implementation issues. We learned several important lessons about facilitating nationwide expansion of a new service into an existing FP program and related integration of the service into existing 
FP support systems. Likewise, as we monitored the process of scale up we learned several important lessons about designing effective M\&E systems that recognize complex environments.

First, it is important to apply a systems lens to monitoring and evaluating the scale-up process and for maintaining a focus on sustained availability of quality services over time. We needed data to inform progress in all subsystems relevant to scale up, such as logistics, policies, demand creation, and provider training. This required multiple sources of data as no individual source of data could accurately reveal all the facets of the situation.

Second, data collected for evaluation purposes do play an important role in monitoring for midcourse corrections during scale up. It is important to not conflate impact evaluation with periodic evaluation, which provides timely information throughout a scale-up process. Secondary data sources such as the SPA were very useful in this case, given limitations of funding for primary M\&E data collection.

Finally, environmental scanning facilitates the ability of the resource team to address political issues related to scale up in a systematic manner. Timely and accurate information about stakeholder opinions, political events, upcoming policy changes, and resource allocations will increase the effectiveness of resource teams to support the scale-up process.

\begin{abstract}
Acknowledgments: The authors thank the Honorable Agnes Binagwaho, the Rwanda Minister of Health, and Corrine Karema, the Head of Division in the Rwanda Biomedical Center. This work could not have been done without their support. The Institute for Reproductive Health, Georgetown University, Washington, DC, supported Standard Days Method scale-up integration efforts and the preparation of this manuscript under cooperative agreement \#GPO-A-00-07-00003-00 with the United States Agency for International Development (USAID) The views expressed by the authors do not necessarily reflect the views or policies of USAID or Georgetown University.
\end{abstract}

Competing Interests: Victoria Jennings is an inventor of the patented CycleBeads technology, owned by Georgetown University, which was at the time of this writing the employer of authors Susan Igras, Irit Sinai, Victoria Jennings, and Rebecka Lundgren. Victoria Jennings is related to the owner of Cycle Technologies, which has an exclusive license from Georgetown University for commercialization of the technology. Victoria Jennings has no financial relationship to and receives no income from Cycle Technologies. Neither she nor the other authors (Rebecka Lundgren, Irit Sinai, Marie Mukabatsinda, Fidele Ngabo, or Susan lgras) receive, nor will they receive, royalties or other income related to the licensed technology.

\section{REFERENCES}

1. World DataBank [Internet]. Washington (DC): World Bank Group. c2014 - [cited 2013 Aug 1.] Available from hitp://databank. worldbank.org/ddp/home.do

2. Institut National de la Statistique du Rwanda (INSR); ORC Macro. Rwanda demographic and health survey 2005. Calverton (MD):
ORC Macro; 2006. Co-published by INSR. Available from: http:// dhsprogram.com/pubs/pdf/FR183/FR183.pdf

3. Arévalo $M$, Jennings $V$, Sinai I. Efficacy of a new method of family planning: the Standard Days Method. Contraception. 2002;65(5): 333-338. Medline

4. Gribble JN, Lundgren RI, Velasquez C, Anastasi EE. Being strategic about contraceptive introduction: the experience of the Standard Days Method@. Contraception. 2008;77(3):147-154. CrossRef. Medline

5. World Health Organization Department of Reproductive Health and Research (WHO/RHR); Johns Hopkins Bloomberg School of Public Health/Center for Communication Programs (CCP), INFO Project. Family planning: a global handbook for providers (2008 update). Baltimore: CCP; 2008. Co-published by WHO/RHR.

6. Simmons R, Fajans P. Contraceptive introduction reconsidered: a new methodology for policy and program development. J Womens Health. 1999;8(2): 163-173. CrossRef. Medline

7. Simmons R, Fajans $P$, Ghiron L, editors. Introduction. In: Simmons R, Fajans $P$, Ghiron L, editors. Scaling up health service delivery from pilot innovations to policies and programmes. Geneva: World Health Organization; 2007. p. vii-xviii. Available from: http://whqlibdoc. who.int/publications/2007/9789241563512_eng.pdf

8. Hardee K, Balogh S, Villinski MT. Three countries' experience with Norplant introduction. Health Policy Plan. 1997;12(3): 199-213. CrossRef. Medline

9. Fajans P, Simmons R, Ghiron L. Helping public sector health systems innovate: the strategic approach to strengthening reproductive health policies and programs. Am J Public Health. 2006;96(3): 435-440. CrossRef. Medline

10. Simmons R, Fajans P, Ghiron L, editors. Scaling up health service delivery from pilot innovations to policies and programmes. Geneva: World Health Organization; 2007. Available from: http:// whqlibdoc.who.int/publications/2007/9789241563512_eng.pdf

11. World Health Organization (WHO); ExpandNet. Nine steps for developing a scaling-up strategy. Geneva: WHO; 2010. Available from: http://whqlibdoc.who.int/publications/2010/ 9789241500319_eng.pdf?ua=1

12. Simmons R, Shiffman J. Scaling up health service innovations: a framework for action. In: Simmons R, Fajans P, Ghiron L, editors. Scaling up health service delivery: from pilot innovations to policies and programmes. Geneva: World Health Organization; 2007. p. 1-30. Available from: http://whqlibdoc.who.int/publications/ 2007/9789241563512_eng.pdf

13. Rogers EM. Diffusion of innovations. 5th edition. New York: Simon \& Schuster; 2003.

14. Blair C, Sinai I, Mukabatsinda M, Muramutsa F. Introducing the Standard Days Method: expanding family planning options in Rwanda. Afr J Reprod Health. 2007; 1 1 (2): 60-68. CrossRef. Medline

15. National Institute of Statistics (NIS) [Rwanda]; Ministry of Health $(\mathrm{MOH})$ [Rwanda]; Macro International Inc. Rwanda service provision assessment survey 2007. Calverton (MD): Macro International Inc; 2008. Co-published by NIS and MOH. Available from: http://dhsprogram.com/pubs/pdf/SPA15/SPA15.pdf

16. Ministry of Health $(\mathrm{MOH})$ [Rwanda]; National Institute of Statistics of Rwanda (NISR); ICF Macro. Rwanda interim demographic and health survey 2007-08. Calverton (MD): ICF Macro; 2009. Co-published by $\mathrm{MOH}$ and NISR. Available from: http:// dhsprogram.com/pubs/pdf/FR215/FR215.pdf

17. León FR, Arévalo M, Lundgren R, Jennings $V$, Huapaya A, Panfichi R. Four criteria to evaluate providers' service-delivery response to new contraceptive introduction. Eval Rev. 2007;31 (4): 364-390.

CrossRef. Medline 
18. Amendezo E. Family planning focal point supervision evaluation in Rwanda. Washington (DC): Institute for Reproductive Health; 2013

19. Morrison JL. Environmental scanning. In: Whitely MA, Portes JD, Fenske $\mathrm{RH}$, editors. A primer for new institutional researchers. Tallahassee (FL): Association for Institutional Research; 1992. p.86-99. Available from: http://horizon.unc.edu/courses/papers/ enviroscan/default.html

20. Sinai I, Lundgren RI, Gribble JN. Continued use of the Standard Days Method(R). J Fam Plann Reprod Health Care. 2012;38(3): 150-156. CrossRef. Medline

Peer Reviewed

Received: 2013 Nov 9; Accepted: 2014 Mar 31; First Published Online: 2014 May 4

Cite this article as: Igras S, Sinai I, Mukabatsinda M, Ngabo F, Jennings V, Lundgren R. Systems approach to monitoring and evaluation guides scale up of the Standard Days Method of family planning in Rwanda. Glob Health Sci Pract. 2014;2(2):234-244. http://dx.doi.org/10.9745/GHSP-D-1300165

(C) Igras et al. This is an open-access article distributed under the terms of the Creative Commons Attribution License, which permits unrestricted use distribution, and reproduction in any medium, provided the original author and source are properly cited. To view a copy of this license, visit http:// creativecommons.org/licenses/by/3.0/ 